\title{
Modelo para determinar la existencia del fenómeno denominado Burbuja Inmobiliaria en el Perú
}

\author{
Model to determine the existence of the phenomenon denominated Burbuja Inmobiliaria in Peru
}

\author{
Carlos Alberto Guerrero López ${ }^{1}$
}

\begin{abstract}
Resumen
El objetivo de la presente investigación fue evaluar si en el Perú se forma una burbuja hipotecaria, o si solo el valor de los precios de las viviendas se encuentra a un dinamismo de BOOM Inmobiliario, este análisis se hace para la década constituida por el período 2004 al 2015. Se analizaron los factores de demanda del sector inmobiliario, identificando si son variables económicas explicativas del dinamismo de los precios del metro cuadrado de los inmuebles en el Perú durante el período indicado; y se examinó si el comportamiento de los precios fue explicado por factores especulativos de los agentes financieros y/u hogares, durante dicho período. Para ello, se elaboró un modelo con variables que expliquen los componentes de la variable precio de viviendas; tales como IPC, tasa de crédito hipotecario del sistema bancario en moneda nacional, en moneda extranjera, tipo de cambio crédito hipotecario en moneda nacional, extranjera y salario real. Es así que a través de diferentes test estadístico se llega a la conclusión que para dicho período el sector inmobiliario en el Perú está en un escenario de Burbuja Inmobiliario; debido a que el precio de las viviendas no está siendo explicado por sus fundamentos económicos.
\end{abstract}

Palabras claves: expectativas inmobiliarias; boom inmobiliario; demanda inmobiliaria; tasa de referencia; salario; tipo de cambio; crédito hipotecario; tasa de interés de crédito hipotecario; análisis de expectativas.

\begin{abstract}
The objective of the present investigation was to evaluate if a mortgage bubble is formed in Peru, or if only the value of housing prices is at a BOOM Real Estate dynamism, this analysis is made for the decade constituted by the 2004 period to 2015. The demand factors of the real estate sector were analyzed, identifying if they are economic variables that explain the dynamism of the prices of square meters of real estate in Peru during the period indicated; and it was examined if the behavior of the prices was explained by speculative factors of the financial agents and / or households, during said period. To do this, a model was developed with variables that explain the components of the housing price variable; such as IPC, mortgage loan rate of the banking system in national currency, in foreign currency, mortgage exchange rate in national, foreign currency and real salary. Thus, through different statistical tests we reach the conclusion that for this period the real estate sector in Peru is in a scenario of Real Estate Bubble; because the price of housing is not being explained by its economic fundamentals.
\end{abstract}

Key words: real estate expectations; real estate boom; real estate demand; reference rate; salary; exchange rate; mortgage credit; mortgage interest rate; expectations analysis.

\section{Introducción}

La vivienda es considerada un bien básico en la economía, por permitir a los hogares tener un techo donde vivir; en las últimas décadas, su uso ha variado hacia un negocio que genera rentabilidad, que permite a estos hogares tener liquidez y obtener ganancias, vale decir como un activo de refugio o un bien de inversión (Gutierrez y Delclóx, 2017).

Según Nenova (2010), los bancos vienen cambiando su papel de financiamiento de crédito de corto plazo hacia los de largo plazo, otorgando en economías estables a sus clientes un menor riesgo por tipo de interés y comisiones accesibles. Diversos especialistas que no pertenecen al sector financiero expresan distintas opiniones respecto a la situación en la que se encuentra el sector inmobiliario; unos afirman que se encuentra en una Burbuja Inmobiliaria, mientras que otros consideran que no se encuentra dentro de una Burbuja Inmobiliaria; ello es debido a que los precios han subido y se debe a la caída del crecimiento económico sufrido en los últimos años en el sector de construcción, perdió su dinamismo que repercutió en el sector inmobiliario.

El presente estudio trató de buscar una explicación basándose en un estudio del sector entre los años 2004 al 2015, analizó si el precio de los inmuebles fue explicado por sus fundamentos económicos de demanda o por expectativas de crecimiento irracional por parte de los 
agentes económicos. Se sabe que en todos los países, el sistema financiero se constituye en un factor clave en el crecimiento de la economía, a través de su función de intermediación financiera que le permite convertir sus depósitos en créditos. Schumpeterm (2003), King y Levine (1993) y Nenova (2010) señalan que este sistema, al proveer de créditos a los hogares para financiar la adquisición de un inmueble, es considerado procíclico dado que guarda una relación directa con el ciclo económico de la economía de un país. Es decir, al aumentar la economía se incrementa el acceso al crédito y al disminuir la economía se obtiene un menor acceso a dichos créditos.

Se debe tener en consideración que la crisis subprime se caracterizó por características completamente ajenas a las viviendas en desarrollo de mercados: bajas tasas de interés, altos ratios de préstamos a valor, una explosión de siniestros dentro del sistema financiero, la extensión a clientes con mal crédito y la complejidad del proceso de titularización y los modelos de valoración subyacentes (Cevallos et al., 2013).

La inflación es una variable a considerar, según como lo afirma Cecchetti (2003), los precios de los activos están basados en expectativas de inflación de la economía, siendo así debido a que los hogares ante subidas de las tasas de inflación tienen menor disponibilidad de liquidez para poder adquirir activos de más valor, tales como viviendas.

Otra variable la constituye la tasa de interés de crédito hipotecario realizadas a través de la política monetaria de los Bancos Centrales; es así que un aumento de las tasas de la política monetaria lleva a que los pagos que realizan las instituciones financieras sean mayores por sus créditos interbancarios, ocasionando una disminución de la liquidez para préstamos a los hogares; generando con ello que disminuyan los créditos hipotecarios y aumenten las tasas de interés correspondientes. Las expectativas según Goodfriend (2005), es otra variable, pues la tasa de política monetaria afecta los precios de la vivienda por medio de las expectativas que tienen los agentes económicos sobre el crecimiento económico y ello repercute en las expectativas de futuros flujos de los agentes económicos que les generan expectativas en su rentabilidad. Además, Hott \& Jokippi (2012) mencionan que las tasas de interés, en el corto plazo, tienen un efecto inverso en el precio de las viviendas y sobre todo en la sobrevaloración de estos precios. Esto se da, debido a que, ante una disminución de las tasas hipotecarias, los hogares tendrán la posibilidad de acceder a mayores créditos hipotecarios, porque los intereses serán menores, impulsando así al aumento de la demanda de viviendas, y a un crecimiento en los precios de las mismas.

En Estados Unidos entre los años 2002 y 2005 se originó una disminución continua de las tasas de interés realizadas por la Federal Reserve System (FED), lo que generó la caída de las tasas de interés a largo plazo, dado el efecto directo indicado anteriormente. La disminución de las tasas de política monetaria, que son de corto plazo, llevaron a que las de largo plazo disminuyan también (Glaese et al., 2010).
Otra variable son los ingresos o salarios de las familias que permite cubrir los pagos de los créditos obtenidos. Además, estos ingresos ayudan a que las familias tengan, ante un nivel alto de ingresos, un mayor acceso al crédito hipotecario en el sistema financiero. Es por ello que los salarios de las familias se constituyen en una variable explicativa del sector inmobiliario. Esta relación entre precios de la vivienda e ingresos reflejan la eficiencia relativa de los mercados inmobiliarios (Banco Mundial, 1994).

Finalmente, el tipo de cambio, que es la relación de intercambio entre dos divisas; debido a que ante una apreciación de la moneda extranjera, mayor será el pago del préstamo y de los intereses hipotecarios, dado que se paga más, generando con ello un mayor costo y un riesgo de no pago Hunter et al. (2003).

\section{Materiales y métodos}

Boom Inmobiliario, los precios de los inmuebles al ser activos utilizados se ven afectados por cambios en la oferta y demanda del mercado inmobiliario y ello repercute en los precios de los mismos. Así, según lo explica Montalvo (2008), existe un crecimiento sostenible del valor de los inmuebles, que se produce específicamente a factores de oferta y demanda del activo inmobiliario. De esta manera sostiene Nenova (2010) que los factores de crecimiento desde la perspectiva de la oferta, el limitado acceso a la tierra y problemas de regulación urbana y desde la perspectiva de la demanda afirma el aumento demográfico de los países, que necesitan cubrir su necesidad básica de vivienda.

Las economías al pasar por ciclos económicos, se presenta situaciones de asimetrías de la información conlleva a niveles altos en los precios de las viviendas, que no son explicadas por sus fundamentos económicos de oferta y demanda. Gutierrez \& Delclóx (2017) afirmaron que las expectativas por el crecimiento de un país se constituyen en un aspecto fundamental para que se manifieste el boom inmobiliario debido a las expectativas de las variables macroeconómicas futuras son favorables.

Expectativas Inmobiliarias, muchas veces las expectativas del crecimiento de los precios de las viviendas en el futuro, de los agentes económicos son expectativas irreales; debido a que estos agentes utilizan a las viviendas como activos de inversión a largo plazo, Gutierrez \& Declóx (2017) al considerar estos activos como una oportunidad para generar rentabilidades futuras, llevan a que los precios de las viviendas suban por presión de la demanda; y son impulsadas no por variables de oferta y demanda sino por expectativas de crecimiento. Estas expectativas son generadas en base al análisis y pensamientos económicos erróneos, Montalvo (2008) define a falacias populares, tales como que a) el precio de las viviendas siempre se mantendrán en niveles altos, porque siempre van a ser demandados por los hogares b) por escasez de tierras por urbanizar el precio del área por metro cuadrado va a tender 
al alza, c) el precio de las viviendas son bajas en relación a los de la región, d) el costo de transacción al ser altos no permitirá la especulación e) el pagar la hipoteca siempre va a permitir que la vivienda sea propia, f) la inversión en vivienda siempre generará mayor rentabilidad que otro activo financiero, obviando que cada economía es distinta y tiene diferentes variables macroeconómicas, el no pago y factores externos de la economía que pueden generar disminución en el trabajo y aumento de desempleo, inestabilidad del sistema financiero y de la economía del país.

Burbuja Inmobiliaria, Montalvo (2003), Case \& Shiller (2004) y Hyramna (1980) definen como un crecimiento rápido y no sostenible de los precios durante un periodo de tiempo. Es rápido porque aumenta a un nivel que no puede ser explicada por fundamentos económicos de oferta y demanda, esto debido a que los agentes proyectan que el valor del activo aumentara y por tal motivo, en búsqueda de obtener rentabilidades futuras, prefieren invertir en ello para poder revenderlos a mayor precio en el futuro, ante la irreal idea de que los precios siempre subirán (Evanoff et al., 2012).

Kindleberger y Aliber (2005) indican la existencia de fases de una burbuja financiera: choque positivo de la economía, que impulsa que las expectativas de los inversionistas suban al ver que pueden obtener futuras rentabilidades, seguido de un boom en sistema financiero, lo anterior genera un aumento de préstamos otorgados por el sistema financiero, ello por la necesidad de los agentes de obtener liquidez para la inversión; proceso de nueva visión, los gobiernos impulsan el crecimiento del sector inmobiliario generando una etapa de euforia, los agentes obtienen rentabilidades y las realizan, observando que sus flujos futuros aumentaran; luego aparece la noción real, algunos inversionistas con mayor información del mercado se dan cuenta que el precio del activo está sobrevaluado, y empiezan a vender este, creando así una sobreoferta que impulsa a la caída abrupta del precio del activo. Finalmente aparece, la etapa de colapso, que es la repercusión en la economía, la burbuja afecta al sector y también al país, generando una búsqueda de soluciones para disminuir sus efectos de la crisis (Evanoff et al., 2012). La crisis subprime tiene características completamente ajenas al desarrollo del mercado de vivienda: bajas tasas de interés, altos ratios de préstamos a valor, una explosión de siniestros dentro del sistema financiero, la extensión a clientes con mal crédito y la complejidad del proceso de titulización y los modelos de valoración y valoración subyacentes (Nenova, 2010).

\section{Metodología}

El estudio pretende demostrar el comportamiento del sector inmobiliario peruano, y el dinamismo de los precios de las viviendas, que han ido en aumento en los últimos años. Hecho que ha motivado a que investigadores revisen el comportamiento del sector, con el fin de evitar caer en burbujas financieras del sector, tal y como las acontecidas en Estados Unidos y España, cuyas crisis fueron las que más impactaron a nivel mundial.

Se utilizó el modelo de cointegración, de FMOLS Mínimos Cuadrado Ordinarios Completamente Modificados (Philllips \& Hansen, 1989), buscando proporcionar a los modelos de cointegración regresiones óptimas. El método permite tener un estimador insesgado, robusto y eficiente en los resultados estadísticos de las regresiones. Este modelo corrige los efectos de correlación serial en los errores, así como la endogeneidad de los regresores, derivados de una relación de cointegración. Además, se utilizó el estadístico tau de Phillps Ouliaris, con la finalidad de contrastar si existe o no cointegración en el largo plazo entre las variables de demanda y el valor de los precios de la vivienda.

Este modelo fue utilizado en estudios como el de Orrego (2014) que explicó que el dinamismo de los precios de las viviendas de los distritos de Lima entre el período 1998 y 2013, están siendo explicados por el comportamiento de la oferta y demanda del sector inmobiliario y que estos cointegran con el dinamismo de los precios.

Vilchez (2015), dentro de su análisis del sector inmobiliario utiliza el método de cointegración para identificar si el valor de los precios de las viviendas en el Perú está siendo explicado por sus fundamentos de oferta y demanda, llegando a través del método de cointegración de Johanse a la conclusión que las variables de demanda y oferta cointegran con el valor de los precios de los inmuebles en el Perú.

A nivel internacional tenemos los estudios sobre la burbuja inmobiliaria realizados por Hoong (2016) sobre Malasia; y de Cubeddu et al. (2012) sobre Chile, Colombia, México y Perú. Para la aplicación del modelo de cointegración, por medio del modelo de estimación FOMLS, se utilizaron las siguientes variables indicadas en la Tabla 1:

Para estimar el modelo de cointegración, por el método de FMOLS, se utilizaron, datos con periodicidad trimestral, desde el período del primer trimestre del año 2004 hasta el cuarto trimestre del 2015. Se evalúó como variable el precio por metro cuadrado de los inmuebles de los distritos de La Molina, Miraflores, San Borja, San isidro y Surco, distritos de la Provincia de Lima; y como variables explicativas se toma variables macroeconómicas y financieras relacionadas a la demanda del sector inmobiliario, como la inflación, la tasa de referencia, las tasas de crédito hipotecario del sistema bancario en soles y en dólares, el tipo de cambio interbancario promedio de compra y venta, el crédito hipotecario en soles y en dólares, y el salario promedio, todas variables de tipo nominal. Al respecto los estudios de Orrego (2014), Cubeddu et al., (2012) y Vilchez (2015), utilizaron variables semejantes llegando a la conclusión que las variables mencionadas eran variables explicativas del precio por metro cuadrado de los inmuebles y que cointegran en el largo plazo. 
Tabla 1. Descripción de Variables

\begin{tabular}{|c|c|c|c|c|}
\hline Definición & Periodicidad & Tipo & Unidades & Fuente \\
\hline Precio & Trimestral & Nominal & $\begin{array}{c}\text { Nuevos soles } \\
\text { corrientes }\end{array}$ & BCRP \\
\hline IPC Lima & Trimestral & Nominal & Porcentaje & INEI, BCRP \\
\hline Tasa de referencia & Trimestral & Nominal & Porcentaje & BCRP \\
\hline Tasa de crédito hipotecaria del sistema bancario en $\mathrm{M} / \mathrm{N}$ & Trimestral & Nominal & Porcentaje & SBS \\
\hline Tasa de crédito hipotecaria del sistema bancario en $\mathrm{M} / \mathrm{E}$ & Trimestral & Nominal & Porcentaje & SBS \\
\hline TC Nominal Interbancarios Precio Compra/Venta -Promedio Mensual & Trimestral & Nominal & S/. por US\$ & BCRP, SBS \\
\hline Crédito del sector Privado de Sociedades de Deposito- Saldo- hipotecario M/N & Trimestral & Nominal & Millones de S/. & BCRP \\
\hline Crédito del sector Privado de Sociedades de Deposito- Saldo- hipotecario M/E & Trimestral & Nominal & Miles de US \$ & BCRP \\
\hline Salario real & Trimestral & Nominal & Soles corrientes & BCRP \\
\hline
\end{tabular}

\section{Modelo Económico Propuesto}

Para la determinación del modelo se parte de una ecuación básica en la cual el crédito hipotecario, es una de las fuentes principales que utilizaron los hogares para conseguir o adquirir una vivienda; así como también los ahorros y las remesas enviadas del exterior por familiares residentes y no residentes. Un mayor crédito hipotecario permitirá que las familias tengan mayor disponibilidad en adquirir una vivienda, impulsando este crecimiento a una mayor demanda y así se generará un aumento de los precios de las viviendas. Por ello, se analizará el flujo de los créditos hipotecarios en soles y dólares emitidos por las entidades financieras para identificar si el crecimiento de la misma tiene el mismo efecto en el precio de las viviendas en el Perú, y se toma por separado los créditos hipotecarios por tipo de moneda, para establecer la implicancia del tipo de cambio en el comportamiento de los precios de las viviendas.

\section{Precio $_{t}=\beta_{1}+\beta_{2}$ Credhipsol $_{t}+\beta_{3}$ Credhipdol $_{t}+\varepsilon_{t}$}

Donde: Precio $_{t}=$ Precio por metro cuadrado de los inmuebles; Credhipsol $=$ Crédito Hipotecario en Soles; Credhipdol ${ }_{t}=$ Crédito Hipotecario en Dólares; $\boldsymbol{\varepsilon}_{\mathrm{t}}=$ Término de error.

Este modelo se elabora basanándonos en los trabajos realizados por Orrego (2014), Arshanapallli y Nelson (2008) y Hoong (2016), que tienen como objetivo identificar si el comportamiento del crédito hipotecario total cointegra con el valor de los precios por metro cuadrado de los inmuebles.

Para reducir la variación de los valores extremos y no extremos de las variables, con el fin de mejorar la fiabilidad de los resultados de los test estadístico, se establecerá el logaritmo de las variables.

$\log$ Precio $_{t}=\beta_{1}+\beta_{2} \log$ Credhipsol $_{t}+\beta_{3} \log _{\text {Credhipdol }}+\varepsilon_{t}$

Donde: $\log$ Precio $_{t}=$ Logaritmo del Precio por metro cuadrado de los inmuebles; $\log$ Credhipsol $=$ Logaritmo del Crédito Hipotecario en Soles; logCredhipdol $=$ Logaritmo del Crédito Hipotecario en Dólares; $\boldsymbol{\varepsilon}_{\mathbf{t}}=$ Término de error.
A este modelo se le agregará otra variable, las tasas de crédito hipotecario del sistema financiero, tanto en soles como en dólares, ello debido a que forman parte de la demanda del sector inmobiliario, por el efecto que generen a los hogares, las variaciones de las mismas. Al aumentar ellas, los hogares se verán obligados a pagar más por sus préstamos hipotecarios, contrayendo de este modo la demanda de las viviendas, generando posteriormente una disminución en los precios de las viviendas. Por lo que se hace necesario analizar si el comportamiento de las tasas hipotecarias son variables explicativas en el largo plazo del precio de las viviendas. Acá se diferencia las tasas hipotecarias por cada moneda para aislar las implicancias que ocasiona el tipo de cambio en el comportamiento de los precios de las viviendas.

Precio $_{\mathrm{t}}=\beta_{1}+\beta_{2}$ Credhipsol $_{\mathrm{t}}+\beta_{3}$ Credhipdol $_{\mathrm{t}}+\beta_{4}$ Tasahipsol $_{t}+\beta_{5}$ Tasahipdol $_{t}+\varepsilon_{t}$

Donde: Precio $_{\mathrm{t}}=$ Precio por metro cuadrado de los inmuebles; Credhipsol $=$ Crédito Hipotecario en Soles; Credhipdol $_{t}=$ Crédito Hipotecario en Dólares; Tasahipsol ${ }_{t}$ $=$ Tasa de Interés Hipotecario en Soles; Tasahipdol $=$ Tasa de Interés Hipotecario en Dólares

$\boldsymbol{\varepsilon}_{\mathbf{t}}=$ Término de error.

Este modelo se basa en los estudios realizados por Vilchez (2015); Cubeddu et al (2012); Hoong (2016), quienes estudiaron el comportamiento de las tasas de interés de créditos hipotecarios con el valor de los precios por metro cuadrado de los inmuebles. Tambien, tomamos el logaritmo a los diferentes términos de la ecuación salvo a las tasas de interés hipotecario, debido a que en ellos no es necesario reducir las variaciones de los valores extremos.

$\log$ Precio $_{t}=\beta_{1}+\beta_{2} \log$ Credhipsol $_{t}+\beta_{3} \log _{\text {Credhipdol }}+\beta_{4}$ Tasahipsol $_{t}+\beta_{5}$ Tasahipdol $_{t}+\varepsilon_{t}$

Donde: $\log$ Precio $_{t}=$ Logaritomo del Precio por metro cuadrado de los inmuebles; logCredhipsol $=$ Crédito Hipotecario en Soles; $\log \mathrm{Credhipdol}_{\mathrm{t}}=$ Crédito Hipotecario en Dólares; Tasahipsol $=$ Tasa de Interés Hipotecario en Soles; Tasahipdol ${ }_{t}=$ Tasa de Interés Hipotecario en Dólares; $\boldsymbol{\varepsilon}_{\mathrm{t}}=$ Término de error 
Finalmente, se adicionan al modelo las variables salario promedio, tipo de cambio promedio de compra y venta, tasa de referencia de la política monetaria y la inflación como variables que explican las variaciones de los precios en las viviendas. Esto siguiendo los estudios de Cubeddu et al. (2012), Borraz et al. (2012), Guerrero (2013) y Hoong (2016).

Precio $_{t}=\beta_{1}+\beta_{2}$ Credhipsol $_{t}+\beta_{3}$ Credhipdol $_{t}+\beta_{4}$ Tasahipsol $_{t}$ $+\beta_{5}$ Tasahipdol $_{t}+\beta_{6}$ Tasaref $_{t}+\beta_{7}$ Tipocambio $_{t}+\beta_{8}$ Salario $_{\mathrm{t}}+\beta_{9}$ Inflación $_{\mathrm{t}}+\varepsilon_{\mathrm{t}}$

Donde: Precio $_{t}=$ Precio por metro cuadrado de los inmuebles; Credhipsol ${ }_{t}=$ Crédito Hipotecario en Soles; Credhipdol $_{t}=$ Crédito Hipotecario en Dólares; Tasahipsol $_{t}$ $=$ Tasa de Interés Hipotecario en Soles; Tasahipdol ${ }_{t}=$ Tasa de Interés Hipotecario en Dólares; Tasaref $f_{t}=$ Tasa de Referencia de la Política Monetaria; Tipocambio ${ }_{t}=$ Tipo de Cambio Promedio de Compra y Venta; Salario $_{t}=$ Salario Promedio; Inflación ${ }_{t}=$ Inflación; $\boldsymbol{\varepsilon}_{\mathbf{t}}=$ Término de error.

Igualmente se reduce la variación de los valores extremos y no extremos de las variables con el fin de mejorar la fiabilidad de los resultados de los test estadístico, por lo que se establecerá el logaritmo para las variables, a excepción de la tasa de interés hipotecario en soles, la tasa de interés hipotecario en dólares, la tasa de referencia de la Política Monetaria y la inflación, por no requerir de ello.

LogPrecio $_{t}=\beta_{1}+\beta_{2}$ LogCredhipsol $_{t}+\beta_{3}$ LogCredhipdol $_{t}$ $+\beta_{4}$ Tasahipsol $_{t}+\beta_{5}$ Tasahipdol $_{t}+\beta_{6}$ Tasaref $_{t}+\beta_{7}$ LogTipocambio $_{t}+\beta_{8}$ LogSalario $_{t}+\beta_{9}$ Inflación $_{t}+\varepsilon_{t}$

Donde: $\log _{\text {Precio }}=$ Precio por metro cuadrado de los inmuebles; LogCredhipsol ${ }_{t}=$ Crédito Hipotecario en Soles; $\operatorname{LogCredhipdol}_{\mathrm{t}}=$ Crédito Hipotecario en Dólares; Tasahipsol $_{\mathrm{t}}=$ Tasa de Interés Hipotecario en Soles; Tasahipdol $_{t}=$ Tasa de Interés Hipotecario en Dólares; Tasaref $_{t}=$ Tasa de Referencia de la Política Monetaria; LogTipocambio $_{t}=$ Tipo de Cambio Promedio de Compra y Venta; $\operatorname{LogSalario}_{\mathrm{t}}=$ Salario Promedio; Inflación $\mathrm{t}_{\mathrm{t}}=$ Inflación; $\boldsymbol{\varepsilon}_{\mathrm{t}}=$ Término de error.

\section{Resultados y Discusión}

Por medio del análisis econométrico se contrastó la primera hipótesis específica que indica que los factores de demanda del sector inmobiliario no son variables económicas explicativas del dinamismo de los precios por metro cuadrado de los inmuebles en el Perú entre el periodo 2004 y 2015. Para ello, se estimó por el método FMOLS, para analizar si las variables de demanda cointegran en el largo plazo el dinamismo de los precios por metro cuadrado de las viviendas.

Para evitar no tener una regresión espuria que no permite tener un análisis de alto nivel de significancia estadística y económico, se realizan pruebas de raíz unitaria que permite establecer el nivel de integración de las variables de demanda del sector hipotecario, así como del predio por metro cuadrado de las viviendas.

Es así que en el Tabla 3 se observa el nivel de diferencia de cada variable del modelo económico final en logaritmos, por medio del test de raíz unitaria de Dickey Fuller Aumentado. Para este test se usó el criterio Schwarz. Además, el nivel de significancia para rechazar la hipótesis nula de raíz unitaria que se usó fue de $10 \%$.

Las variables del precio por metro cuadrado de las viviendas, los créditos en soles, créditos en dólares, la tasa de interés hipotecario en soles y dolares, la inflación, el salario y el tipo de cambio se han incluido para el test la tendencia y el intercepto, más no para la tasa de referencia que se incluyó solo en la interjerción, como se muestra en la Tabla 2.

En la Tabla 3, la inflación, el salario y la tasa de referencia son intercepto cero, I(0), lo que significa que son estacionarias a nivel. El crédito hipotecario en soles, el precio por metro cuadrado de las viviendas, las tasas de interés hipotecarias en soles y dólares y el tipo de cambio son I(1) o estacionarias en primera diferencia. La única variable que es $\mathrm{I}(2)$ o estacionaria en segunda diferencia es del crédito hipotecario en dólares. En el análisis de cointegración, por el Método FMOLS, es necesario utilizar las variables que están integradas en primera diferencia, I(1).

Es así que en el modelo no se incluirá el crédito hipotecario en dólares, la inflación, el salario y la tasa de referencia.

Tabla 2. Análisis de Dickey Fuller Aumentada

\begin{tabular}{|c|c|c|c|c|c|}
\hline \multirow[t]{4}{*}{ Variable } & \multirow[t]{4}{*}{ Modelo } & \multicolumn{3}{|c|}{ Augemented Dickey Fuller } & \multirow{4}{*}{$\overline{\mathrm{I}(\mathrm{d})}$} \\
\hline & & Niveles & Primera & Segunda & \\
\hline & & Prob. & diferencia & liferencia & \\
\hline & & & Prob. & Prob. & \\
\hline logcredhipdol & Ty C & 0.9922 & 0.4104 & 0.0000 & $\mathrm{I}(2)$ \\
\hline logcredhipsol & Ty $\mathrm{C}$ & 0.8883 & 0.0949 & ----- & $\mathrm{I}(1)$ \\
\hline Inflación & $\mathrm{T}$ y C & 0.0212 & ----- & ----- & $\mathrm{I}(0)$ \\
\hline logPrecio & $\mathrm{T}$ y $\mathrm{C}$ & 0.1432 & 0.0000 & ----- & $\mathrm{I}(1)$ \\
\hline logsalario & Ty C & 0.0250 & ----- & ----- & $\mathrm{I}(0)$ \\
\hline tasahipsol & $\mathrm{T}$ y $\mathrm{C}$ & 0.4453 & 0.0000 & ----- & $\mathrm{I}(1)$ \\
\hline tasahipdol & $\mathrm{T}$ y C & 0.5385 & 0.0001 & ----- & $\mathrm{I}(1)$ \\
\hline tasaref & $\mathrm{C}$ & 0.0085 & ----- & ----- & $\mathrm{I}(0)$ \\
\hline logtcambio & $\mathrm{T}$ y $\mathrm{C}$ & 0.9997 & 0.0037 & ----- & I(1) \\
\hline
\end{tabular}

Tabla 3. Regresión FMOLS de cuatro (4) variables explicativas

\begin{tabular}{ccccc}
\hline Variable & Coefficient & Std. Error & t- statistic & Prob \\
\hline logcredhipsol & 0.270642 & 0.033558 & 8.064894 & 0 \\
logtc & 0.093093 & 0.414449 & 0.22462 & 0.8234 \\
tasacredhipdol & -12.10184 & 4.062769 & -2.978716 & 0.0048 \\
tasacredhipsol & 6.60343 & 1.977375 & 3.339493 & 0.0018 \\
C & 6.075822 & 0.883768 & 6.874906 & 0 \\
\hline
\end{tabular}

En el Tabla 4, se observa la regresión de cointegración por el Método FMOLS y se muestra que el crédito hipotecario en soles, las tasas hipotecarias en soles, las tasas hipotecarias en dólares son significativas en el modelo de 
cointegración, a un nivel de significancia de $5 \%$, más no el tipo de cambio. Por lo que en el modelo no se incluye a esta variable como explicativa de precio por metro cuadrado de las viviendas en el Perú.

Tabla 4. Regresión FMOLS de créditos hipotecarios en soles y tasa hipotecaria en dólares

\begin{tabular}{ccccc}
\hline Variable & Coefficient & Std. Error & t- statistic & Prob \\
\hline logcredhipsol & 0.19366 & 0.039741 & 4.8731 & 0 \\
tasacredhipdol & -14.83737 & 6.185558 & -2.398711 & 0.0208 \\
C & 7.763456 & 0.833636 & 9.312768 & 0 \\
R-squared & 0.877636 & Mean dependent var & 8.001212 \\
Ajusted & 0.872074 & S.D. dependent var & 0.459714 \\
R-squared & & & \\
$\begin{array}{c}\text { S.E. of } \\
\text { regression }\end{array}$ & 0.164425 & Sum squared resid & 1.189566 \\
\hline
\end{tabular}

Tabla 5. Test de Cointegración

\begin{tabular}{lcc}
\hline & Value & Prob.* \\
\hline Engle-Granger tau-statistic & -2.341573 & 0.5773 \\
Engle-Granger z- statistic & -9.588153 & 0.5907 \\
& Value & Prob.* \\
& & 0.667 \\
Phillis-Ouliairs tau-statistic & -2.156273 & 0.745 \\
Phillis-Ouliairs z-statistic & -7.435667 & 0.756 \\
*MacKinnon(1996) p-values. & &
\end{tabular}

La estimación de cointegración, por el método FMOLS, que se muestra en el Tabla 4, expresa que las variables explicativas de la demanda del sector inmobiliario, crédito hipotecario en soles y tasa de crédito hipótecarios en dólares, son significativas a un nivel de significancia del 5\% con un R cuadrado ajustado del 87,2074\%.

Luego se realizan el test de cointegración de Engle y Granger y de Philips Ouliaris mostradas en el Tabla 5 observándose que los estadísticos tau, que ayudara a verificar si los residuos de la regresión son estacionarios en nivel, son de 0,577 y 0,6670 , respectivamente. Estos estadísticos al 5\% de nivel de significancia aceptan la hipótesis nula de que las series no están cointegradas en el largo plazo.

Tabla 6. Test de Cointegración

\begin{tabular}{lcc}
\hline & Value & Prob.* \\
\hline Engle-Granger tau-statistic & -2.341573 & 0.5773 \\
Engle-Granger z- statistic & -9.588153 & 0.5907 \\
\hline & Value & Prob.* \\
\hline Phillis-Ouliairs tau-statistic & -2.156273 & 0.667 \\
Phillis-Ouliairs z-statistic & -7.435667 & 0.745 \\
\hline *MacKinnon(1996) p-values. & & \\
Fuente: Elaboración propia & &
\end{tabular}

La variable crédito hipotecario en soles es significativo, a un $5 \%$ de significancia, así como su efecto positivo en el dinamismo de los precios de las viviendas, con un $\mathrm{R}$ cuadrado ajustado de $84,7703 \%$. Además al igual que el modelo anterior que incluía a la tasa hipotecaria en dólares, se observa en la Tabla 6, que el test de cointegración de Engle y Granger y de Phillips Ouliaris no rechazan la hipótesis nula de no cointegración de las variables, obteniéndose estadísticos tau de 0.7579 y 0.7416 , respectivamente, al $5 \%$ de significancia.

Tabla 7. Regresión MCO del crédito hipotecario en soles y cuatro rezagos de precios de viviendas

\begin{tabular}{ccccc}
\hline Variable & Coefficient & Std. Error & t- statistic & Prob \\
\hline logcredhipsol & 0.054175 & 0.01723 & 3.144278 & 0.0032 \\
logprecio(-1) & 0.611816 & 0.160747 & 3.80607 & 0.0005 \\
logprecio(-2) & -0.030248 & 0.185158 & -0.163362 & 0.8711 \\
logprecio(-3) & 0.180638 & 0.184198 & 0.980675 & 0.333 \\
logprecio(-4) & 0.094026 & 0.14957 & 0.628643 & 0.5333 \\
C & 0.73795 & 0.287293 & 2.568634 & 0.0143 \\
\hline
\end{tabular}

En la Tabla 7, se observa la regresión por método de estimación MCO y en ella se observa que el crédito hipotecario en soles, junto al primer rezago de los precios de las viviendas muestran una significancia al 5\%, al rechazar la hipótesis nula de no significancia, así como signos positivos en el efecto que tienen en el precio de las viviendas. También se muestra que el segundo, tercero y cuarto rezago del precio de las viviendas son no significativos, porque no se rechaza la hipótesis nula de no significancia al $5 \%$.

Si eliminamos el segundo, tercero y cuarto rezago al modelo estimado por MCO, las variables crédito hipotecario en soles y el primer rezago de los precios son variables explicativas de la regresión, a un nivel de significancia del $5 \%$. Los resultados que se observan en el Tabla 8 , muestran un efecto positivo del crédito hipotecario en soles con el precio de las viviendas, esto porque ante un mayor nivel del crédito hipotecario, significa mayor demanda de viviendas, generando un aumento de los precios de los inmuebles. Por este efecto es mínimo en relación al efecto mostrado por el primer rezago del precio, que indica que los precios de las viviendas están siendo explicadas en mayor nivel por el precio del trimestre anterior en mayor relación a los créditos hipotecarios en soles que muestran un coeficiente de 0.04 , indicando que un crecimiento del $100 \%$ de este tipo de crédito afectará solo un $4 \%$ en el precio de las viviendas.

Tabla 8. Regresión MCO del crédito hipotecario en soles y el primer rezago de los precios de viviendas

\begin{tabular}{ccccc}
\hline Variable & Coefficient & Std. Error & t- statistic & Prob \\
\hline logcredhipsol & 0.040033 & 0.01288 & 3.10821 & 0.0033 \\
logprecio(-1) & 0.882035 & 0.044689 & 19.73714 & 0.0000 \\
C & 0.635102 & 0.262597 & 2.418539 & 0.0198 \\
\hline
\end{tabular}

Tabla 9. Test de Robustez del Modelo MCO

\begin{tabular}{llll}
\hline \multicolumn{4}{c}{ Breusch-Godfrey Serial Correlation LM Test } \\
\hline F-static & 1.889904 & Prob. F(4,40) & 0.1311 \\
Obs*R-squared & 7.470665 & Prob Chi-Square(4) & 0.1130 \\
\hline \multicolumn{4}{c}{ Heteroskedasticity Test Breusch-Pagan-Godfrey } \\
\hline F-static & 1.537417 & Prob. F(4,40) & 0.2263 \\
Obs*R-squared & 3.069946 & Prob Chi-Square(2) & 0.2155 \\
Scaled explained SS & 1.978263 & Prob Chi-Square(2) & 0.3719 \\
\hline
\end{tabular}


En este punto es necesario comprobar la robustez del modelo, es decir identificar si los regresores y datos estadísticos de la regresión son válidos para el análisis de los resultados. Para analizar tal robustez es necesario realizar el diagnostico de los resultados. Para analizar esa robustez, es necesario realizar el diagnostico de los residuos analizando el test de normalidad correlación serial y heterocedasticidad, que muestra una probabilidad de 0.2269 , valor mayor al $5 \%$ de significancia, rechazándose así la hipótesis nula de que los residuos presentan varianza constante. Ello demostró que la regresión por MCO realizado es robusta y que sus valores estadísticos son factibles para el análisis a realizar si el rezago de los precios explica el dinamismo del mismo.

En la Tabla 9 se muestra los resultados de las tres pruebas, en ella se observa que en el test de normalidad no se rechaza la hipótesis nula que los residuos se distribuyen normalmente, a un 5\% de significancia. También se observa el test de correlación serial de Breusch Godfrey donde se analiza desde el cuarto rezago de los residuos, teniendo como resultado una probabilidad de 0,1311 , valor mayor al 5\% de significancia, no rechazándose así la hipótesis nula de que los residuos no están correlacionados. Y además se tiene el test de heterocedasticifad.

\section{Conclusiones}

El sector inmobiliario en el Perú, entre los períodos 2004 y 2015, se encuentró en un escenario de Burbuja Inmobiliaria

El dinamismo del precio de las viviendas no es explicado por sus fundamentos económicos, debido a la inexistencia de una relación en el largo plazo de las variables de demanda con el precio por metro cuadrado de las viviendas. Aún, cuando en efecto, en el período analizado, el crédito hipotecario en soles ha mostrado un crecimiento continuo, este aumento no explica el crecimiento más elevado de los precios de las viviendas, en el largo plazo.

La estabilidad de la política económica en el Perú, tanto en niveles de inflación y tasas de interés, está generando que los agentes económicos y financieros, además de los hogares vean en el sector inmobiliario como una oportunidad de obtener rentabilidades, por medio de ventas futuras de las viviendas, ante la expectativa generada de un crecimiento de los precios de estas. Dado que el primer rezago de los precios de las viviendas, explica e mayor nivel el crecimiento de los precios, en relación a los créditos hipotecarios

Se corroboró que las tasas de interés de créditos hipotecarios, en el largo plazo, no tienen un efecto significativo, tanto estadístico como económico, en el precio de las viviendas en el Perú. Las tasas de interés de créditos hipotecarios en soles mostró una relevancia positiva con el crecimiento de los precios, siendo económicamente inviable este efecto, debido a que un aumento de las tasas de interés contrae a la demanda y por efecto el también nivel de precios, y viceversa. Además, la tasa en dólares mostró una relevancia positiva y significativa, pero siendo mínimo su efecto en el precio de las viviendas.
Se recomienda aplicar en el Perú un mayor nivel de regulación financiera del sector hipotecario, con el fin de frenar el crecimiento de la burbuja inmobiliaria en el Perú así como el efecto negativo en el sistema financiero que repercutirá en la economía peruana. Aun cuando no existe, en el largo plazo, un efecto significativo de las tasas hipotecarias en el precio de las viviendas, estas deben ser monitoreadas por la SBS. Debido a que a menores tasas de interés en el sector inmobiliario en el corto plazo, repercutirá en un alza en los precios de las viviendas. Y si a esto último se le adiciona que los niveles de ingresos y salarios de los hogares no crecen al mismo nivel, pueden generar un mayor nivel de riesgo crediticio en el sistema financiero. Finalmente, se recomienda para futuras investigaciones levantar la limitación del presente estudio de considerar los precios de sectores altos de Lima que en promedio poseen un mayor nivel de ingresos al promedio peruano.

\section{Literatura citada}

Arshanapalli, B. and Nelson, W. 2008. A cointegration test to verify the housing Bubble. Indiana University Northest. 2, 35-42.

Banco Mundial. 1994. Vivienda: Un entorno propicio para el mercado habitacional, Washington D.C.

Borraz, Licandro y Ponce. 2012. Precios de Viviendas: una metodología para evaluar desvíos respecto a sus fundamentos. Banco Central del Uruguay. 16. 8-21.

Case, K. \& Shiller, R. 2004. Is there a buble in the housing market?. Cowles Foundation Paper $N^{\circ}$ 1089. Cowles Foundation for Research in Economics. Yale University Box 208281. New Haven, Connecticut 06520-8281. http://cowles.econ.yale.edu/

Cecchetti, S. G. 2003. Asset Prices in a Flexible Infationn targeting. Framework Ohio State University and National Bureau of Economic Research Hans Genberg, Chapter 30.

Cevallos, F.; Didier, T.; Hevia, C. y Schumecler, S. 2013. Respuestas de política a la crisis financiera global: ¿Qué hicieron diferentes economías emergentes? Banco Central de Reserva del Perú, Revista Estudios Económicos 24: 27-43.

Cubeddu, L.; Tovar, C. and Tsounta, E. 2012. Latin America: Vulnerabities under Construction?. IMF Working Paper, 193, 8-13.

Evanoff, D.; Kaufman, G. \& Malliaris, A. G. 2012. Asset Price bubbles: What are causes, consequences and public policy options?.Chicago FED Letter, Essays on Issues The Federal Reserve Bank of Chicago November 2012, No 304.

Glaese, E.; Gottlieb, J. \& and Gyourko, J. 2010. Can Cheap Credit Explain the Housing Boom? The National Bureau of Economic Research-NBER Working Paper No. 16230. Issued in July 2010, NBER Program(s): EFG.

Goodfriend, M. 2005. Interest rate Policy Should Not 
React Directly to Asset Prices. Federal reservw Bank of Richmond, Chapter 31.

Guerrero, Y. 2013. Burbuja Inmobiliaria en el Perú, periodo 2000-2013 (Tesis se pregrado). Universidad Privada Antenor Orrego. Trujillo, Perú.

Gutierrez, A. y Delclós, X. 2017. Geografia de la Crisis Inmobiliaria en Cataluña: Una lectura a partir de los desahucios por ejecución hipotecaria. Scrpta Nova, revista Electronica de Geografia y Ciencias sociales. Universidad de Barcelona. Vol. XXi, Num. 557.

Hyramna, M. 1980. Money, financial markets, and the coherence of a market economy. Journal of Post Keynesian Economics/Fall Vol III (1).

Hott, C. and Jokippi, T. 2012. Housing bubbles and interest rates. Working Pper of the Swiss National Bank.

Hoong, W. 2016. Detecting Malaysian Housing Bublles. American Journal of Applied Sciences 281-288.

Hunter, W.; Kaufman, K. \& Pomerleano, M. 2003. Asset Price Bubbles The Implications for Monetary, Regulatory, and International Policies.,

King, R. y Levine, R. 1993. Finance and growth: Shumpeter might be right, The Quarterly Journal of Economics, 108 (103): 717-737.

Kindleberger and Aliber. 2005. Manias, Panics, and Crashes A History of Financial Crises, Seventh Edition, Palgrave Macmillan UK.

Montalvo, J.G. 2008. De la quimera inmobiliaria al colapso financiero. Crónica de un desenlace anunciado. Antoni Bosch, Barcelona.

Nenova, T. 2010. Expanding housing finance to the underserved in South Asia: market review and forward agenda. The World Bank. Agenda Washington D.C.

Orrego, F. 2014. Precios de viviendas en Lima. Banco Central de Reserva del Perú, 8, 1-13.

Phillips, P. and Hansen, B. 1989. Statical Inference in Instrumental Variables Regression whith I (1) Processes. Review of Economic Studies 57,99-125.

Schumpeter, J. 2003. The Theory of economic development, The European Heritage in Economics and the Social Sciences, 1:61-116.

Vilchez, D. 2015. Assesing house price dynamics in Lima. Graduate Institute of Intenational and Development Studies, 9, 1-22. 